\title{
Analysis of the Effect of Hormones on Opposite-Sex Twin Attitudes
}

\author{
E.M. Miller ${ }^{1}$, N. Martin ${ }^{2}$ \\ ${ }^{1}$ Department of Economics and Finance, University of New Orleans; ${ }^{2}$ Queensland Institute of \\ Medical Research
}

\begin{abstract}
Numerous reports in the literature suggest that hormones may transfer from one fetus to another, in humans as in animals. In a large sample of over seven thousand Australian adult twins, it was found that opposite-sex females showed a statistically significant tendency to hold more masculine attitudes than did same-sex female twins. This may be due to post-natal social interaction, but could also be caused by the transfer of testosterone from the male to the female fetus in opposite-sex twins.
\end{abstract}

Key words: Opposite-sex twins, Hormones, Testosterone, Attitudes, Australia

\section{INTRODUCTION}

It is now well established that hormones can transfer from one fetus to another in experimental animals [10], [23], [36]. This effect was first found by a researcher when he discovered that female mice adjacent to male mice in the uterus had larger ano-genital distances at birth, and later exhibited more masculine behavior than females adjacent to females. This was attributed to testosterone from the male fetuses affecting the female fetuses, since the effect on ano-genital distance did not appear when the mothers were treated with antiandrogens.

One possibile mechanism for such hormone transfer was suggested when it was shown that testosterone could diffuse across amniotic membranes in rats [9]. Another possible mechanism was indicated by evidence suggesting that the masculinizing effects on female rats of uterine position were probably due to transfer of testosterone through the maternal blood circulation [21]. These effects were observed in female fetuses downstream from males, but not in those upstream, with supporting evidence also being provided which showed that a male's presence on the uterine horn's caudal side had a more critical influence than contiguity per se [26]. 
Many aspects of behavior and morphology have been shown to be affected by uterine position. Twenty-five years ago, two authors showed that blood serum and amniotic fluid concentrations of testosterone for female mice depended on uterine position [37], as did the attractiveness of female mice to males, and infanticide and parental behavior in males [35]. Male gerbils who were adjacent to two males had higher blood levels of testosterone than males who were between two females [2].

These effects are often large in magnitude. In mice, the effect of intra-uterine position was great enough for females located between two males (and therefore exposed to high levels of testosterone) to have body weights that were in the male range for all but the last few days of the experiment [16]. These masculinized females actually weighed more than the feminized males who had been situated between two females in the uterus.

In the same experiments, female mice located between two males (with higher testosterone levels) were less active than females located between females [15]. The effect was great enough for females who had been affected by testosterone from male fetuses to resemble male mice more in their behavior than did the unexposed females mice who had been located between females in-utero. Not only can hormones transfer, but enough can transfer to have effects comparable in magnitude to the normal male/female differences.

\section{Can hormone transfer occur in human twins?}

It has been noted that in pregnant humans much of the estrogen in the maternal blood is of fetal origin, suggesting that similar effects occur in humans [35]. A subsequent study by two prominent neurologists formed similar hypotheses [11], although the authors did not develop these in a follow-up study.

There are several reasons for believing testosterone and other sex hormones can permeate the human placenta. Experiments with monkeys have shown that the injection of testosterone into pregnant mothers produces behavioral and morphological changes in the offspring, including the growth of a penis in female offspring, for example [13]. Such results indicate that testosterone is able pass through the placenta from the mother to the fetus in primates, and it seems reasonable to suppose that the process can also occur in humans.

The extensive literature on maternal and fetal hormones has as its underlying assumption that maternal, fetal, and placental hormonal states affect each other, either by direct transfer of hormones, or by transfer of precursors [28], [34]. In the late 1980s, another author also stated that "... steroids readily cross the placenta". More recently, it has been shown that maternal blood testosterone level varies with the sex of the fetus being carried [22]. These authors conclude that "as a consequence of a maternal-fetal gradient, unbound testosterone crosses the placenta from the male fetus toward the maternal circulation, whereas the opposite direction applies to a female fetus" (p. 53). Thus testosterone apears able to pass from fetus to mother (human), and from mother to fetus (at least in monkeys, guinea pigs, and rats). Thus it seems possibile for a male twin to be able to affect his twin sister's testosterone level.

Higher levels of plasma estrogen have been reported in mothers of twins than were found in mothers of singletons [32]. The authors speculate that "The high oestrogen 
levels in twin pregnancy may have been a result of the four fetal adrenal glands producing an excess of precursor DHEAS and two fetal livers producing enough 16-hydoxylase for formation of these steroids" (p. 193). In the mid-1980s, a group of workers reported $40 \%$ higher estriol levels in mothers of twins than in mothers of singletons [33]. The fact that these female hormones transfer between the maternal and the fetal circulations makes it more plausible that the structurally similar male hormones could transfer between fetuses.

It has been found that estrogen concentrations in blood and in urine during pregnancy correlate with birthweight [6]. While estrogen might somehow promote fetal growth (or be correlated with a factor that promotes it), the estrogen/birthweight correlation is more simply explained by the fact that the estrogen the fetus produces enters the maternal circulation, and that large fetuses produce more estrogen. Cortisol, which has been shown to be structurally similar to testosterone [29], is believed to transfer from the mother to the fetus. The maternal contribution to fetal cortisol is calculated at between $25 \%$ and $50 \%$, while two authors estimated the maternal contribution to fetal cortisone to be almost $100 \%$ [12]. By injecting radioactive forms into the mother and observing radioactivity in the fetus, cortisol and DHEAS have been shown to transfer from the maternal to the fetal circulation. Of course, if steroids can transfer between maternal and fetal circulations, they should be expected to transfer from one fetus to the other via the mother, and possibly more directly.

Evidence has also been produced to suggest that handedness and sexual orientation in incipient humans can be affected by maternal stress via the mother's stress hormones (adrenaline, cortisol and corticosterone), which reach the fetus, as in the case of experimental animals [7]. If these effects are real, they would provide evidence that hormones structurally similar to sex hormones can pass from the mother to the fetus in humans. Cortisol has a structure which is very similar to testosterone. The evidence that the fetus affects its mother's hormones and vice-versa makes of it quite plausible that one fetus can affect another hormonally. testosterone appears to be the hormone most likely to both transfer between fetuses and to affect fetal development. The fetus is believed to develop into a female, unless acted on by testosterone from a male fetus' testes. Because a human has to be fully male or female to reproduce, evolution would select for high levels of fetal testosterone. The cost in reproductive fitness of providing a large safety margin would be low, while the loss in reproductive fitness of an inadequately masculinized male would be large. However, female fetuses do produce some testosterone in the adrenal glands, and female tissues appear to be affected by such testosterone. If only a small fraction of the testosterone from the male somehow reaches the female, the quantity could be large relative to her own production, and could have an appreciable masculinizing effect.

As opposite-sex twins occur approximately half as often as same-sex twins (representing $35 \%$ of twin births), any disadvantages arising from the transfer of male fetal testosterone to female twins would affect only a small percentage of offspring (perhaps $0.005 \%$ or one in 200), and this would not select against male fetuses producing large amounts of testosterone, or females retaining sensitivity to it. 


\section{Other evidence for hormonal transfer in humans}

Other researchers have reported evidence that humans experience hormone transfer. One study of teeth diameters in human twins found that males generally had larger teeth in the right jaw (consistent with the larger right hemisperes of their brains) [1]. The asymmetry in dental diameters could be used to separate males from females. The author reports: "A linear discriminant function sex-classification rule correctly classified 42 of the 44 same-sex DZ in our sample, but misclassified 12 of our 20 opposite-sex twins $\left(X^{2}{ }_{1}=21.6\right.$, exact $\left.P=3 \times 10^{-7}\right), 8$ of the 10 females, and 4 of the 10 males. A quadratic discriminant function sex-classification rule that correctly classified 123 out of $128 \mathrm{SS}$ twins of both zygosities, misclassified 16 of the 20 opposite-sex DZ twins $\left(\mathrm{X}_{1}{ }_{1}=72.1\right), 7$ of the 10 males, and 9 of the 10 females"' (p. 601).

Hormone transfer between fetuses would seem to offer the most plausible explanation of this phenomenon, whereby opposite-sex females' dental diameters become more masculine, or those of their male cotwins become more feminine.

An unpublished paper used human twins to report the effects of a variety of variables considered relevant to alcohol consumption [38]. It was shown that female behavior did not depend on the sex of the cotwin, while males showed a significant effect for only 1 out of 14 variables. However, for 6 out of 7 tests, the opposite-sex males had reactions that were more feminine. The one statistically significant result in males related to the number of incorrect responses to the motor coordination task. Here the score dropped from 17.7 (Standard error $=1.2$ ) for same-sex DZ males to 12.5 (Standard error $=1.6$ ) for opposite-sex DZ males. The same-sex female values were 12.8 (Standard error = 1.2), versus 9.9 (Standard error $=1.2$ ) for opposite-sex females. Thus, the opposite-sex males have values in the female range. While this may only be a chance result, further research is called for, as its possible effects may be sizeable and in the direction predicted.

A recent study examined sensation-seeking in a sample of 422 British adult twins, including 51 opposite-sex pairs [25]. Age-adjusted sensation-seeking behavior was significantly higher in opposite-sex compared with same-sex females. The increase was statistically significant both for an overall measure, and for subscales measuring disinhibition (interpreted as interest in socially and sexually disinhibited activites), and adventurousness (interpreted as seeking new experiences in a non-conformist way through travel, new intellectual interests or consciousness-altering drugs). Opposite-sex females had elevated scores for thrill and adventure seeking, and for boredom susceptibility. Males had a statistically significant tendency to score higher on all scales. Thus, the opposite-sex females' attitudes were consistently more masculine than those of the samesex females. The results were interpreted as being consistent with hormone transfer. No consistent pattern was found when the same-sex males were compared to those with a female cotwin.

Significantly higher baseline spatial scores on the Vandenberg modification of the Shepard-Metzler Mental Rotations test have been reported for opposite-sex DZ females than for same-sex females [4]. Moreover, opposite-sex females showed a greater improvement over three trials than did the same-sex females. "By the third trial, the scores of these opposite-sex DZ females were not significantly different from those of their twin brothers", although sex differences were otherwise found. Impressively, the effect was large enough to eliminate these sex differences. The authors concluded that "These 
results suggest the possibility that exposure to testosterone in-utero improves spatial ability in females, thus supporting the theory that differences in prenatal exposure to testosterone are at least partially responsible for the gender differences in spatial ability".

An earlier study reported many measures for a sample of twins and matched controls, young children cross-classified by both zygosity and sex [17]. Unfortunately, the study only contained opposite-sex twin pairs, but the effects observed were often in the direction hypothesized in this paper. "The striking divergence noted between the sexes when two groups, uniform in sex, are compared is not apparent in the two sex groups derived from opposite-sex pairs" ' is the author of the study's most revealing statement (p. 163). Unfortunately, since the hypothesis that is the subject of this paper had not yet been developed, the author did not consider hormone transfer as a possible explanation for her results.

\section{British public opinion questionnaire data}

One other study of opinions in opposite-sex twins has been conducted [23]. The data was obtained from a large-scale twin study conducted in London, and summarized in an Appendix [5]. As part of a larger study of 1650 twins in the early $1970 \mathrm{~s}, 60$ questions had been asked, dealing with such topics as sexual behavior, religion, and politics. ${ }^{1}$ For the majority of questions, there was not a statistically significant difference between the answers of opposite- and same-sex twins. For females, in only 16 out of 60 questions did replies show a statistically significant difference $(p<0.05)$. For 14 of these 16 questions, the difference took the form of the female twins answering the question more as males did. In only two questions were the answers more "feminine", and in both cases the magnitude of the sex-effect was small. For males, in only 5 out of the 60 questions was there a statistically significant difference in their replies. However, in all five of these cases, the male twins who had shared a womb with a female cotwin had more feminine attitudes. Although the difference in males' responses was statistically significant in considerably fewer questions ( 5 out of 60 ) than that of females (16 ont of 60 ), the results of the questionnaire suggest that the sex of cotwins is important.

As a measure of the sex effect, the differences between the sexes in twins' replies to the questions were calculated (by subtracting the average female from the average male score). For each item, the difference in average scores between the female opposite-sex and same-sex twins was calculated. This measured the effect on a female twin of her cotwin's sex. The two difference scores, (sex and sex of cotwin) were correlated. They had a correlation of 0.54 , a value which is far too high to have occurred randomly. Adult females with twin brothers replied to the questionnaire in a more masculine way than those with sisters. This result is consistent with hormones transferring in the womb.

When a similar calculation was done for the males surveyed by the questionnaire, the correlation was -0.22 . The negative sign indicates that males with a female cotwin shifted towards the feminine pattern of replies. However, the effect was not quite statistically significant.

\footnotetext{
${ }^{1}$ See [5] Appendix for the 60 questions that constitute the Public Opinion Inventory.
} 


\section{METHODS AND RESULTS}

\section{Australian twin data}

Given the results of the above study, replication in another sample was called for. Fortunately, a suitable data-set was available. As part of another study [20], a sample of Australian adult twins, including 905 opposite-sex pairs, 1982 female pairs, and 916 male pairs had been given a 50 -item version of the Wilson-Patterson conservatism scale. This comprises a series of one word items to which the subjects rate their agreement by circling "yes", "'?", or "no". The data is the same that has been used previously by other authors [5], [20] to show that family resemblance in social attitudes is caused more by genetic influences than by shared environmental influences (culture).

For the purposes of this study, the three possible answers were converted to a numeric scale by scoring "yes" as 0 , "?" as 0.5 , and " no" as 1 . These scores were then crosstabulated by sex and sex of cotwin. For most of the items, males and females differed in their responses. A measure of this sex difference was calculated by subtracting the female score for the same-sex twins from the male score. This was done for both the samesex and opposite-sex twins. The subtraction was algebraic, retaining the sign of the differences. The magnitude and direction of the sex differences were very similar in the same-sex and in the opposite-sex pairs.

A statistically significant difference was frequently found in the attitudes of the twins, which depended on the sex of the opposite twin. For females, the average difference in the absolute value of the opinions between members of opposite-sex and same-sex pairs was 0.024 . This average difference was 0.015 for males. Some of these differences only reflect sampling variability. As there were fewer male than female pairs, if the differences between opposite-and same-sex twins were only due to sampling varia-

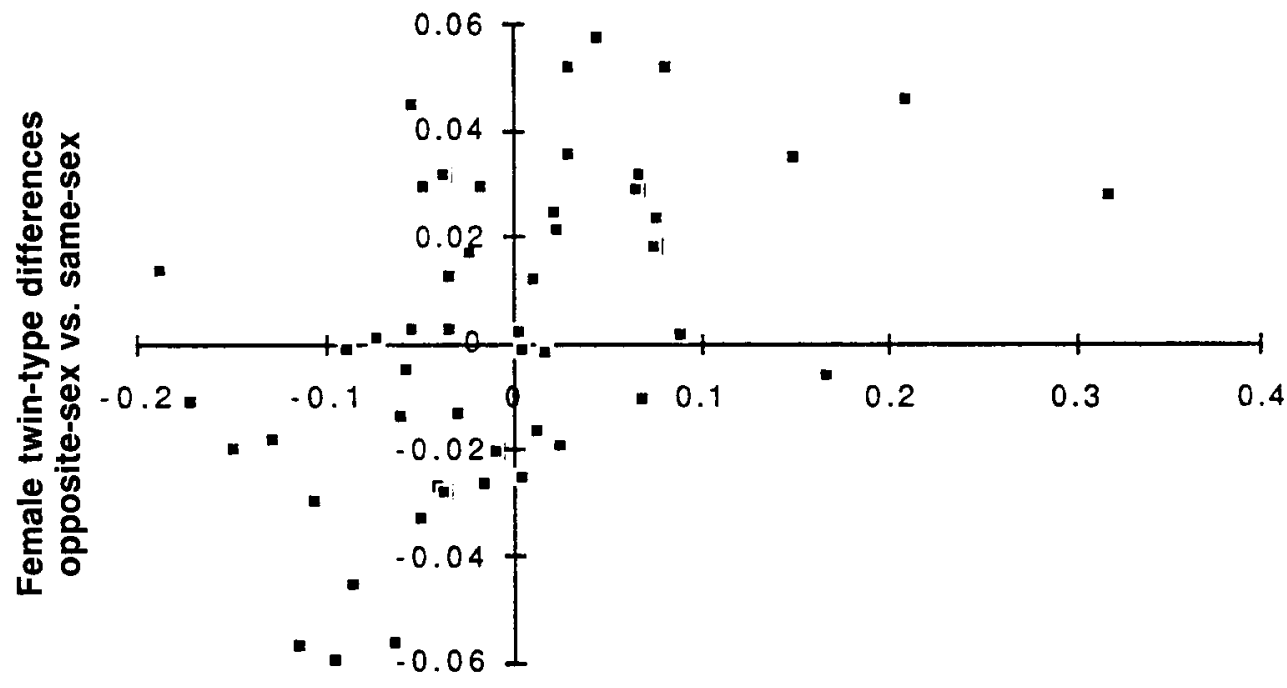

Sex differences in same-sex twins

Fig. 1. Australian female twin attitudes. 
bility, they would be greater among the males. However, they are greater among the females. This suggests that the sex of a cotwin influences females' opinions.

Indeed, the male answers in the opposite- and same-sex pairs showed no systematic differences. Opposite-sex males replies' were almost as masculine as those of the samesex males. ${ }^{2}$ The correlation coefficient between the difference in male twin attitudes and the sex difference was 0.21 , explaining less than $5 \%$ of the variance. This was not a statistically significant effect.

The most striking finding can be seen in the Figure. The $x$-axis shows the sex differences on each question for the same-sex twins with the questions where males had the higher scores on the right. Ones where females scored higher are to the left. The $y-$ axis shows how big a difference (with sign retained) the sex of the female's twin made. If females with brothers scored higher, the difference is positive and above the $x$-axis. As can be seen, there is a strong tendency for female twins with twin brothers to give more masculine answers.

It was decided to measure the sex differences from the same-sex twin answers to avoid any attenuation of the greater similarity of the opposite sex twins. In a regression of the difference in female attitudes between twin types on the sex difference, the coefficient for the sex difference was 0.16 . The regression coefficient is interpreted as the opposite-sex female attitudes moving an average of $16 \%$ of the way toward the typical male's attitudes. The standard error of the regression coefficient was 0.04 , making it highly unlikely that the correlation is due to chance. The regression explains $24 \%$ of the variance in the difference between same-sex and opposite-sex female twin opinions, corresponding to a correlation coefficient of 0.49 .

\section{DISCUSSION}

As has been shown, the basic pattern of results from this large Australian sample rather closely resembles the results from the London twins questionnaire [23]. Two studies conducted at opposite ends of the world with different instruments produced similar results. A hormonal explanation for the sex-of-twin effect requires accepting the joint hypothesis that hormones both affect opinions and transfer between twin fetuses. If opposite-sex females become more masculine in their opinions, but opposite-sex males do not become more feminine, the effects seem likely to be caused by transfer of a male hormone, probably testosterone. The basic mammalian pattern is female [10], in that a fetus develops as a female, unless exposed to testosterone from the fetal testes. It is plausible that the brain contains testosterone receptors such that after exposure to testosterone, it can easily acquire masculine beliefs and adopt masculine patterns of behavior. This would explain both sex differences and (with testosterone transfer) the masculinization of the opposite-sex females' attitudes. Furthermore, other evidence exists for such masculinization [11].

The reported observations can be explained by either pre-natal or post-natal effects. Either way, it is necessary to explain why the females show a systematic tendency to shift

\footnotetext{
${ }^{2}$ Because any significant sex differences were virtually always in the same direction in both the sameand opposite-sex twins, there is very little ambiguity in referring to a certain direction as masculine.
} 
their opinions in the masculine direction, while the males do not. A social explanation would have to assume a much stronger tendency for females to change their opinions to agree with those who are emotionally close to them, than for males. Admittedly, such a tendency probably exists. It may even be biological. (The hormonal explanation would be that testosterone affects the females greatly, but that either few female hormones are produced by fetal ovaries, or the male is little affected by any female hormones that may reach him).

\section{Possible socialization effects}

Post-natal socialization is the obvious alternative to pre-natal hormonal effects. Since twins form very close emotional bonds in childhood, in studying childhood attitudes, we would expect to find that one twin exerts a very strong effect on the other twin. Perhaps a brother persuades his twin sister to adopt opinions a little more like his. Likewise, a sister may similarly be able to affect her brother. If females tend to shift their opinions more in the male direction than males do in the female direction, this could explain the relatively greater female than male shift of opinions which the results of the London and the Australian twin surveys show. However, after twins grow up, they marry, leave home, and typically see much less of their twins. The Australian twins studied here were adults, who typically were not living with their twins. Most were married. It is well known that spouses see a lot of each other, and have numerous oportunities to influence one other's opinions. In comparing two women, both married, and both heavily exposed to male views through their husbands, it seems inherently implausible that the extent to which they are influenced by male attitudes should still be much affected by the sex of their cotwin.

The closest analogy to an opposite-sex twin relationship for intensity of feeling is marriage. Spouses' replies to public opinion surveys are known to correlate highly. If the similarity resulted from living together, the longer the couples had been married, the more their opinions would resemble each other. However, the degree of spousal resemblance does not increase with age (a good indicator of length of marriage), which suggests that living with and having extensive contact with someone of the opposite sex may not not shift opinions in the direction of that person's opinions [5].

To discover how plausible a socialization hypothesis was, the literature on sibling effects on each other was examined. Both imitation (siblings resemble each other) and contrast (siblings contrast with one other) hypotheses had been proposed. A study of college students reported that evidence (depending on the item) can be found for both the contrast and the imitation hypotheses among second-born males [19]. A previous study reported significant imitative effects among college females in families with two siblings on the Gough Scale of Psychological Femininity [27]. While the score moved from 23.61 (for females with sisters) to 22.41 (females with brothers), a statistically significant shift, this move was still small in relation to the male-female gap (the females' brothers averaged 15.9). While the sex of a sibling had a statistically significant impact, the brother's femininity score had virtually no impact (correlation of 0.03 ) on his sister's which weighs against the imitative hypothesis. Another study found evidence for imitation effects in sports participation and femininity among college females [18]. After their sur- 
vey of the literature, the authors of a more recent study [8] conclude: "The hypothesis that sex of sib has no general and lasting influence on personality has not been refuted", (p. 175).

The above studies used college students, the easiest population for college professors to study. It should be remembered students are typically still living with their siblings, or have lived with them until very recently. Thus, any effects on students due to the attitudes of their siblings would not yet have been diminished by the separation from siblings that normally follows leaving home. In contrast, typical twins answering the public opinion questions analysed above were adults, who had not lived with their cotwins for many years. If strong and consistent sibling effects have not been demonstrated among those who have recently lived with their siblings, any effects among those who have lived apart from their siblings for many years (typically as a result of marriage) would be expected to be weaker.

\section{Research implications}

The authors interpret the results of the above study of Australian twin attitudes as supporting the hypothesis that hormones transfer between fetuses, especially when intepreted in light of the findings of other studies in the literature. However, there is clearly further research to be done. Direct tests of the hormone transfer hypothesis should be made. Comparisons of the hormonel levels in twins' amniotic fluid should be made. Direct assays of hormone levels in stillborn twins should be made. While cord blood analyses are possible, the maximum difference between the sexes in amniotic fluid testosterone concentrations occur well before birth. Thus, comparisons of cord blood at birth may fail to disclose an effect that occurred earlier. Most twin studies have been undertaken as part of genetic or obstetric studies. The probability that hormones transfer provides another reason for studying twins. Opposite-sex and same-sex twins appear to develop in quite different hormonal environments. This provides one of the few ways in which the pre-natal effects of hormones on human behavior can be studied. If done as part of a larger twin study, hormonal effects can often be studied at low cost.

Of course, studying hormonal effects does require including opposite-sex twins in the study design. Unfortunately, most genetically oriented twin studies have been limited to same-sex twins in order to avoid the obvious complications of sex differences. However, there are interesting questions to be answered with opposite-sex twins, and I would urge researchers to study them. In considering the cost of including opposite-sex twins, it should be remembered that as they are always dizygotic, there are no costs for zygosity determination. Also, studies of hormonal effects depend only on knowing the sex of the cotwin. It is not necessary to study pairs of twins. In questionnaire studies, or those based on medical records, all that is necessary is to know is whether a particular individual was an opposite-sex twin, a same-sex twin, or a singleton. A simple question about twin status and the sex of the cotwin can provide useful information. Such information may be wanted for medical reasons (such as the possibility of a tissue transplant, or to use the twins' history as an aid to diagnosis). If such twin information is available in a large data set, say military records, it would be possible to compare twins with nontwins, and opposite- with same-sex twins. 
Research carried out at the end of the 1950s studied twins drafted into the Swedish army during the period 1949-1952 [14]. An interesting peculiarity (crying out for replication) is that on all four subjects for which primary-school marks were available, the opposite-sex male twins did better than either the MZ or the $\mathrm{DZ}$ same-sex twins. Since females would normally be expected to have done better on these subjects, this suggests a hormone transfer effect. If opposite-sex twins develop in an unusual prenatal hormonal environment, it is likely that the patterns of disease they experience may be atypical of other members of their sex. A possible example of such effects is that there are two reports of high rates of ophthalmic defects in opposite-sex twins [17, 31], which one author has discussed elsewhere [24]. If opposite-sex twins are exposed to unusual disease risks, it would obviously be useful to know what these are.

\section{CONCLUSIONS}

Numerous reports in the literature suggest that hormones may transfer from one fetus to another, in humans as in animals. In a large sample of over six thousand Australian adult twins, it was found that opposite-sex females showed a statistically significant tendency to hold more masculine attitudes than did same-sex female twins. This is most likely due to testosterone transfering from the male fetus to the female fetus in oppositesex twins.

Acknowledgement: The authors thank Prof. A.C. Heath of the University of Washington for his cross-tabulation of Australian twins' scores by sex and sex of cotwin for this study.

\section{REFERENCES}

1. Boklage C E (1985): Interactions between same-sex dizygotic fetuses and the assumption of Weinberg difference method epidemiology. Am J of Hum Genet 37:591-605.

2. Clark M M, Vom Saal F S, Galef Jr., B G (1992): Intrauterine positions and testosterone levels of adult male gerbils are correlated. Physiol Behav 51:957-960.

3. Clemens L G (1974): Neuohormonal control of male sexual behavior. In Montagna, W \& Sadler, W A (eds): Reproductive Behavior. New York: Plenum Press.

4. Cole-Harding S, Morstad A L, Wilson J R (1988): Spatial ability in members of opposite-sex twin pairs. Behav Genet 18:710.

5. Eaves L J, Eysenck H J, Martin N G (1989): Genes, Culture, and Personality. London: Academic Press.

6. Ekbom A, Trichopoulos D, Adami H, Hsieh C, Lan S (1992): Evidence of prenatal influences on breast cancer risk. The Lancet 340:1015-1018.

7. Ellis L, Peckham W (1991): Prenatal stress and handedness, J Pre Peri-nat Psychol 6; 2:135-144.

8. Ernst C, Angst J (1983): Birth Order: its Influence on Personality. New York: SpringerVerlag.

9. Fels E, Bosch L R (1971): Effect of prenatal administration of testosterone on ovarian function in rats. Am J Obstet Gynecol 111:964-969.

10. Gandelman R (1992): Psychobiology of Behavioral Development, Ch 3. Oxford: Oxford University Press. 
11. Geschwind N, Galburda A M (1987): Cerebral Lateralization. Cambridge, Mass.: Mit Press, p. 141.

12. Gibson M, Tulchinsky D (1980): The maternal adrenal. In Tulchinsky, D \& Ryan K J (eds): Maternal-fetal Endocrinology. Philadelphia: W B Saunders.

13. Goy R W, Resko J A (1972): Gonadal hormones and behavior of normal and pseudohermaphroditic non-human female primates. In Eastwood EB (ed): Recent Progress in Hormone Research. New York: Academic Press, pp. 707-735.

14. Husen T (1959): Psychological Twin Research Stockholm: Almqvist \& Wiksell.

15. Kinsley C, Miele J, Konen C, Ghiraldi L, Broida J, Svare B (1986): Intrauterine contiguity influences regulatory activity in adult male and female mice. Horm Behav 20:7-12.

16. Kinsley C, Miele J, Wagner C K, Ghiraldi L, Broida J, Svare B (1986): Prior intrauterine position influences body weight in male and female mice. Horm Behav 20:201-211.

17. Koch H L (1966): Twins and twin relations. Chicago: University of Chicago Press.

18. Landers D M (1970): Sibling-sex-status and ordinal position effects on female sport participation and interests. J Soc Psychol 80:247-248.

19. Leventhal G S (1970): Influence of brothers and sisters on sex-role behavior. J Pers Soc Psychol 16:452-465.

20. Martin N G, Eaves L J, Heath A C, Jardine L R, Feingold M, Eysenck H J (1986): Transmission of social attitudes. Proc Nat Acad Sci USA 83:4364-4368.

21. Meisel R L, Ward I L (1981): Fetal female rats are masculinized by male litter mates located caudally in the uterus. Science 213: 239-241.

22. Meulenberg P M M, Hofman J A (1990): Maternal testosterone and fetal sex. J Steroid Biochem Mol Biol 39;1:51-54.

23. Miller E M (1994): Prenatal sex hormone transfer: a reason to study opposite-sex twins. Person In Diff: 17; 4:511-529.

24. Miller E M (1995): Reported myopia in opposite-sex twins: a hormonal hypothesis. Optom Vision Sci $72 ; 1: 34-36$.

25. Resnick S M, Gottesman I I, McGue M (1993): Sensation seeking in opposite-sex twins: an effect of prenatal hormones? Behav Genet 23:323-329.

26. Richmond G, Sachs B J (1984): Further evidence for masculinization of female rates by males located caudally in utero. Horm Behav 18:484-490.

27. Rosenberg B G, Sutton-Smith B (1968): Family interaction effects on masculinity-femininity. J Pers Soc Psychol 8;2:117-120.

28. Schindler A E (1982): Hormones in Human Amniotic Fluid, (Berlin: Springer-Verlag).

29. Schulster D, Burstein S, Cooke BA (1976): Molecular Endocrinology of the Steroid Hormones. London: Wiley \& Sons.

30. Soloman S (1988): The placenta as an endocrine organ. In Knobilm E \& Neil J D (eds): The Physiology of Reproduction. New York: Raven Pressm pp. 2085-2091.

31. Stocks P, Karns M N (1933): A biometric investigation of twins and their brothers and sisters, Pt. II. Annals of Eugenics 5:1-55.

32. TambyRaja R L, Ratnam S S (1981): Plasma steroid changes in twin pregnancies. In Gedda L, Parisi P, Nance W (eds): Twin Research 1: Part A. Twin Biology and Multiple Pregnancy. New York: Alan R Liss Inc, pp. 190-195.

33. Trapp M, Kato K, Bohnet H G, Gerhard I, Weise H C, Leidenberger F (1986): Human placental lactogen and unconjugated estriol concentration in twin pregnancy: monitoring of fetal development in intrauterine growth retardation and single intrauterine fetal death. Am $\mathbf{J}$ Obstet Gynecol 55:1027-31.

34. Tulchinsky D \& Ryan K J (eds) (1980): Maternal-fetal Endocrinology. Phyladelphia: W B Saunders

35. Vom Saal F (1983): Variation in infanticide and parental behavior in male mice due to prior intrauterine proximity to female fetuses: elimination by prenatal stress. Psychol Behav 30:675-681. 
36. Vom Saal F (1989): Sexual differentiation in litter-bearing mammals: influence of sex of adjacent fetuses in utero. J Anim Sci 67:1824-1840.

37. Vom Saal F, Bronson F H (1980): Sexual characteristics of adult female mice are correlated with their blood testosterone levels during prenatal development. Science 208: 597-599.

38. Whitfield J B, Martin N G (1992): Sex differences in alcohol, use, peak concentration, and post-alcohol test performance in humans; a test of the effects of prenatal environment. Manuscript.

Correspondence: Prof. Edward M. Miller, Department of Economics and Finance, University of New Orleans, New Orleans, LA 70148, U.S.A. 